Universidade Tecnológica Federal do Paraná - UTFPR

Campus Ponta Grossa - Paraná - Brasil

ISSN: 1981-3686/v. 04, n. 02: p. 186-197, 2010

DOI:10.3895/S1981-36862010000200007
Revista Brasileira de Tecnologia

Agroindustrial

\title{
ELABORAÇÃO DE BEBIDA ALCOÓLICA FERMENTADA DE JACA (Artocarpus heterophyllus Lam.)
}

\section{ELABORATION A BEVERAGE FERMENTED ALCOHOLIC OF JACKFRUIT (Artocarpus heterophyllus Lam.)}

\author{
Erasto Ferreira de Assis Neto ${ }^{1}$; João Marcos Pinto da Cruz ${ }^{2}$ \\ Adriano César Calandrini Braga ${ }^{3}$; João Hamilton Pinheiro de Souza ${ }^{4}$ \\ ${ }^{1}$ Universidade do Estado do Pará (UEPA), Campus XVIII - Cametá erasto0205@ yahoo.com.br \\ ${ }^{2}$ Universidade do Estado do Pará (UEPA), Campus XVIII - Cametá jmpc1025@ hotmail.com \\ ${ }^{3}$ Universidade do Estado do Pará - UEPA adrianocalandrini@ hotmail.com \\ 4 Universidade do Estado do Pará (UEPA), Campus XVIII - Cametá jhpsouza@yahoo.com.br
}

\section{Resumo}

A jaqueira (Artocarpus heterophyllus) produz a maior de todas as frutas cultivadas e um amplo potencial para ser explorado industrialmente. As bebidas fermentadas de frutas constituem produtos com perspectiva promissora de consumo. O objetivo do presente trabalho foi obter uma bebida alcoólica fermentada de jaca, bem como determinar as características físico-químicas da bebida e avaliar a aceitação da mesma. A bebida alcoólica foi realizada utilizando-se jaca da variedade mole, metabissulfito de sódio e fermento biológico seco (Saccharomyces cerevisae). Na elaboração da bebida, o mosto foi inoculado com o fermento biológico e fermentado entre 25 e 30 ${ }^{\circ} \mathrm{C}$. A sulfitagem foi realizada em $90 \%$ do mosto, sendo o metabissulfito de sódio adicionado na proporção de $200 \mathrm{mg} / \mathrm{L}$. A inoculação foi realizada pelo método de pé-de-cuba. Fez-se o acompanhamento da fermentação realizando análises físico-químicas, que seguiram as Normas do Ministério da Agricultura (BRASIL, 2005), durante um período de 10 dias. No produto final foram encontrados teores de acidez total $(220 \mathrm{meq} / \mathrm{L})$, cinzas $(3,55 \mathrm{~g} / \mathrm{L})$, densidade $(0,99401 \mathrm{~g} / \mathrm{L}) e$ alcoólico $\left(4,1^{\circ} \mathrm{GL}\right)$. Os resultados obtidos da análise sensorial, utilizando o teste aceitabilidade com escala hedonica, demonstraram que a bebida fermentada teve um percentual de aceitação de $61 \%$, apresentando resultados parecidos com o fermentado de outras frutas tropicais. Os resultados da análise sensorial demonstraram que a jaca é apropriada para a produção de bebida alcoólica fermentada, porém, ainda necessita de maiores estudos à condução do processo fermentativo para obtenção do produto dentro de um padrão aceitável pelo mercado.

Palavras-chave: bebida alcoólica; fermentados de frutas; jaca.

\section{Introdução}

O Brasil já é o segundo maior produtor mundial de frutas. Entretanto, há um grande desperdício pós-colheita para algumas culturas, o que, notadamente, gera prejuízos. Apesar de muito ter se feito em prol do crescimento da fruticultura brasileira, ainda falta uma campanha em 
prol do desenvolvimento mais rápido da nossa pomicultura, e outra mostrando o valor da fruta na alimentação humana (GOMES, 2007).

Existe, portanto a necessidade de se desenvolver novos processamentos que permitam a redução das perdas. Uma das maiores vantagens é o fácil cultivo das arvores frutíferas no solo brasileiro, principalmente as mais rústicas, tropicais, que saborosas frutas produzem. E uma das alternativas para que diminua o numero de perdas, é a produção de bebidas alcoólicas a partir de frutas nativas ou daquelas que facilmente se propaguem (DIAS et al, 2003; GOMES, 2007).

A jaqueira (Artocarpus heterophyllus) produz a maior de todas as frutas cultivadas, sendo muito popular em países do Sudeste da Ásia e da África. Porém, encontra-se difundida de forma endêmica em quase todas as regiões tropicais do mundo. É uma árvore de copa irregular que alcança até $25 \mathrm{~m}$ de altura, e apresenta frutos com o peso variando de 3 a $60 \mathrm{~kg}$. Frutas como a jaca, a melancia, o maracujá, entre outras, escondem grandes utilidades culinárias com amplo potencial para serem aperfeiçoadas e industrializadas, com escassez de estudos e pesquisas que destaquem os seus valores nutricionais (PEREIRA et al, 2007; LIMA et al, 2008; CORRÊA, 1984).

A jaca geralmente é consumida in natura, podendo ser utilizada também no preparo de doces ou compotas e na fabricação de um tipo de aguardente (LUNA, 1997). Os "bagos" são constituídos de uma porção comestível chamada polpa, doce e aromática, mais ou menos consistente e que envolve cada semente, podendo estas apresentar de 100 até 500 sementes em fruto (VIEIRA NETO, 2002).

A fermentação alcoólica é um processo que resulta da transformação de açúcares solúveis em etanol. Entre as leveduras mais utilizadas na fermentação alcoólica esta a Saccharomyces cerevisiae, usada na panificação, cervejaria, destilaria, entre outros. Para produzir álcool etílico o mosto (líquido, açucarado apto a fermentar) deverá ter certa concentração de açúcares $\left(16^{\circ}\right.$ a $20^{\circ}$ Brix) e componentes nutritivos (GAVA, 1984).

As bebidas fermentadas de frutas constituem produtos promissores, devido tendência de aceitação em pesquisas de consumo, além de contribuírem para a redução de perdas pós-colheita de frutos perecíveis (SANDHU; JOSHI, 1995). Tradicionalmente, são empregadas uvas e maçãs na obtenção de bebidas fermentadas, porém muitos países, principalmente os europeus, produzem de frutas diferenciadas, como a pêra, a groselha, a framboesa, e a cereja (MUNIZ et al, 2002).

O objetivo do presente trabalho foi a obtenção de uma bebida alcoólica fermentada de jaca, bem como determinar as características físico-químicas da bebida e avaliar a aceitação da mesma junto a provadores. 


\section{Material e Métodos}

A elaboração da bebida alcoólica de jaca (Artocarpus heterophyllus Lam.), foi realizada no Laboratório de Alimentos da Universidade do Estado do Pará (UEPA), Campus XVIII - Cametá. Como matéria prima da elaboração da bebida alcoólica fermentada foi utilizada a jaca da variedade mole. Utilizou-se metabissulfito de sódio e fermento biológico seco (Saccharomyces cerevisiae), da marca Fleischmamn. O processo completo de elaboração da bebida alcoólica fermentada de jaca pode ser observado na Figura 1.

Figura 1 - Fluxograma do processo de elaboração da bebida alcoólica fermentada de jaca.

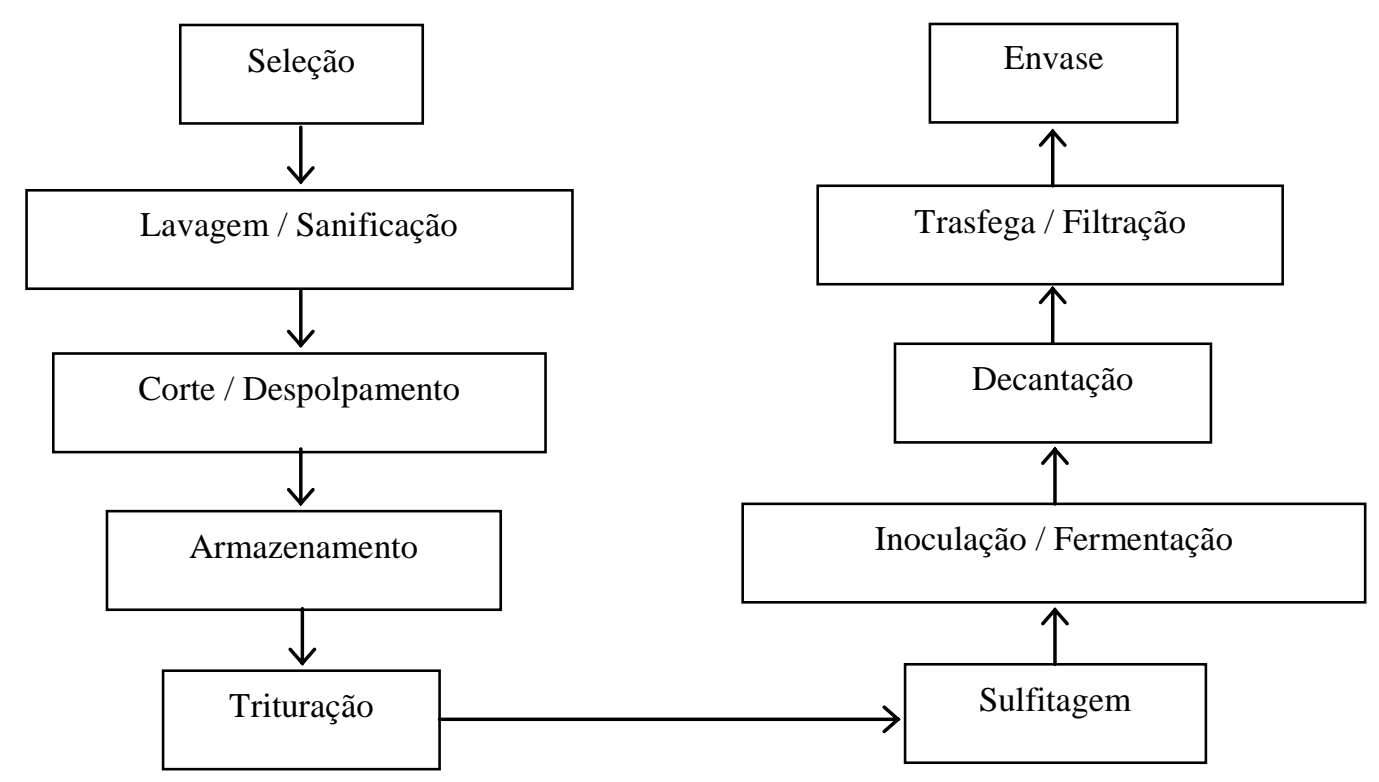

Selecionaram-se os frutos com coloração amarelada e odor forte característico, que indicava um bom estado de maturação. Logo após, lavou-se os frutos individualmente em água corrente, sendo que posteriormente foram imersos em água clorada a $10 \mathrm{ppm}$ durante $15 \mathrm{~min}$. Em seguida foram lavados com água destilada para retirar o resíduo do cloro.

O corte e o despolpamento foram realizados manualmente. As polpas foram acondicionadas em sacos plásticos específicos para polpa e armazenadas a $-18 \pm 1{ }^{\circ} \mathrm{C}$. A trituração foi realizada com um liquidificador, até que a polpa ficasse bem homogênea.

A sulfitagem foi realizada em $90 \%$ do mosto, o agente responsável pela sulfitagem foi o metabissulfito de sódio, onde este foi adicionado na proporção de $200 \mathrm{mg} / \mathrm{L}$ no mosto e colocado sob refrigeração por $24 \mathrm{~h}\left(8 \pm 1{ }^{\circ} \mathrm{C}\right)$. A inoculação foi realizada pelo método de pé-de-cuba, onde foram retirados $10 \%$ do mosto, colocado em balão volumétrico de $1000 \mathrm{~mL}$ e feito uma pasteurização $\left(60^{\circ} \mathrm{C} / 30 \mathrm{~min}\right.$.), deixando esfriar até uma temperatura de $30^{\circ} \mathrm{C}$. Posteriormente, foi 
adicionado o fermento biológico seco na proporção de $200 \mathrm{mg} / \mathrm{L}$ dos $10 \%$ do mosto, permanecendo por $24 \mathrm{~h}$ a temperatura ambiente, sendo então adicionado ao restante do mosto.

Após a inoculação do mosto, foram retiradas algumas amostras onde, fez-se o acompanhamento da fermentação realizando análises físico-químicas, que seguiram as Normas do Ministério da Agricultura (BRASIL, 2005). As análises ocorreram durante o processo até a obtenção da bebida final, num período de 10 dias, e estão descritas abaixo:

- Sólidos solúveis totais ( ${ }^{\circ}$ Brix): Para a determinação de sólidos solúveis foi utilizado o método refratométrico com resultado expresso em ${ }^{\circ} \mathrm{Brix}$;

- Acidez total: A acidez total foi determinada por titulometria com hidróxido de sódio $(\mathrm{NaOH})$ 0,1N utilizando como indicador fenolftaleína. O resultado foi expresso meq/L;

- $\mathrm{pH}$ : determinou-se por medidor de $\mathrm{pH}$ digital portátil modelo da INSTRUTHERM.

Dando continuidade ao processo, a decantação foi realizada sob refrigeração $\left(8 \pm 1^{\circ} \mathrm{C}\right)$ por 24h. Após a decantação o mosto foi retirado do recipiente de PVC, utilizado na fermentação, e colocado para um recipiente de poliestireno.

Após a trasfega o produto foi filtrado com o auxílio de tecidos de algodão e crivo para separação das partículas sólidas. Após a filtração o produto foi colocado em três balões volumétricos de $1000 \mathrm{~mL}$ e submetido a uma pasteurização em banho-maria $\left(60{ }^{\circ} \mathrm{C} / 30 \mathrm{~min}\right.$. $) \mathrm{O}$ produto final foi envasado em garrafas PET de $180 \mathrm{~mL}$.

A caracterização físico-química da bebida foi realizada segundo a metodologia proposta pelo Ministério da Agricultura (BRASIL, 2005). As amostras foram analisadas no LACEN Laboratório Central, Departamento de Análises de Produtos (DAP), localizada no Distrito de Icooaraci - Belém. As análises realizadas foram:

- Densidade: determinada pelo método densimétrico através de picnômetros;

- Acidez total: mesmo procedimento anteriormente descrito na fermentação;

- Teor alcoólico: obtido (\% em volume) pela tabela de conversão de densidade relativa a $20{ }^{\circ} \mathrm{C} / 20^{\circ} \mathrm{C}$ determinada no destilado alcoólico da amostra;

- Cinzas: incineração a temperatura de aproximadamente $550^{\circ} \mathrm{C}$ até peso constante.

A caracterização sensorial da bebida alcoólica fermentada de jaca foi realizada no Laboratório de Alimentos da Universidade do Estado do Pará Campus XVIII - Cametá, onde se aplicou o método sensorial afetivo, teste de aceitabilidade de escala hedônica de nove pontos, onde em seus extremos apresentam os termos gostei muitíssimo (9) de desgostei muitíssimo (1) (FERREIRA, 2000).

$\mathrm{Na}$ avaliação, as amostras foram servidas a 41 provadores não treinados de ambos os sexos e de idades variadas, em recipientes de $50 \mathrm{~mL}$, através de fichas como da Figura 2. 
Figura 2 - Ficha de avaliação para teste de aceitabilidade utilizando escala hedônica de nove pontos.

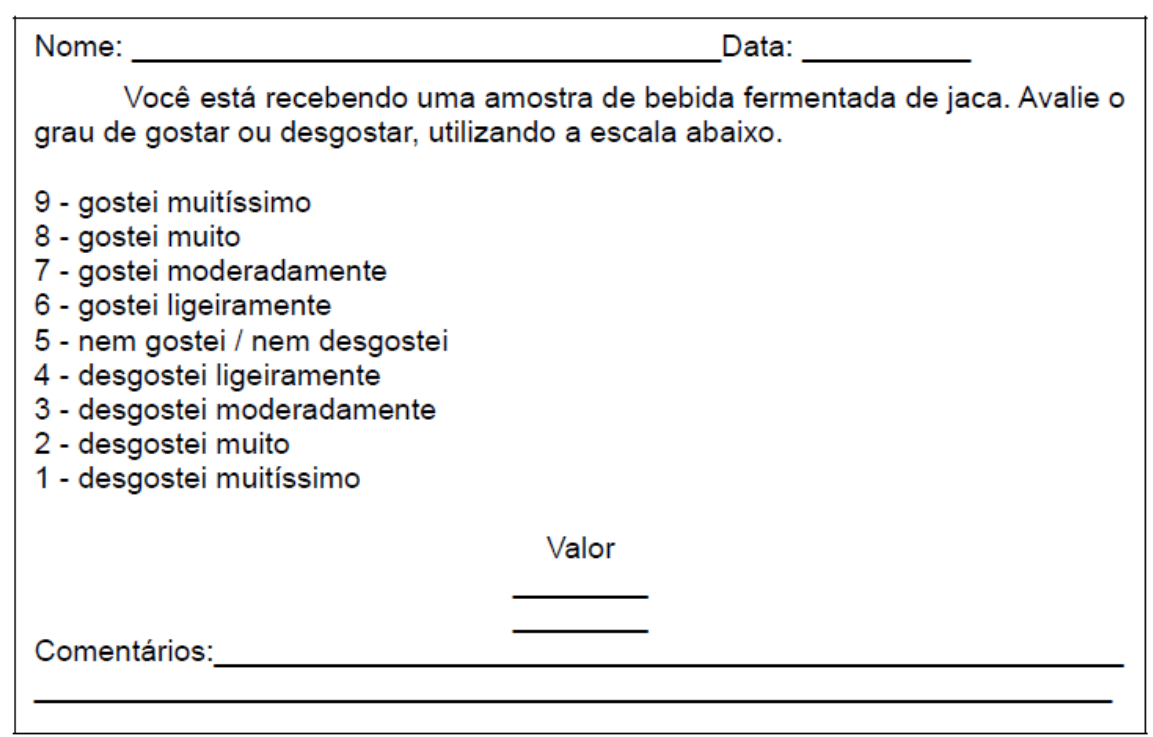

Fonte: FERREIRA, 2000.

\section{Resultados e discussão}

Os resultados da bebida fermentada de jaca foram comparados a trabalhos com outros tipos de fermentados oriundos de frutas tropicais, visto que não há legislação especifica para bebidas fermentadas de frutas tropicais e a lei relata apenas os atributos de graduação alcoólica (BRASIL, 1997).

A bebida fermentada apresentou uma aparência chamativa onde se destacou por apresentar coloração e aroma característico da fruta. A fermentação da bebida teve o acompanhamento dos parâmetros: sólidos solúveis ( $\left.{ }^{\circ} \mathrm{Brix}\right), \mathrm{pH}$, acidez total. $\mathrm{O}$ tempo decorrido foi de dez dias onde foi verificado o andamento do processo fermentativo. A Tabela 1 mostra os valores encontrados durante a fermentação.

Tabela 1 - Valores encontrados durante a fermentação.

\begin{tabular}{cccc}
\hline $\begin{array}{c}\text { Dias de } \\
\text { fermentação }\end{array}$ & $\begin{array}{c}\text { Sólidos solúveis } \\
\text { ('Brix) }\end{array}$ & $\mathbf{p H}$ & $\begin{array}{c}\text { Acidez total } \\
\text { (meq/L) }\end{array}$ \\
\hline 01 & 16,0 & 5,8 & 50 \\
02 & 14,8 & 5,5 & 61 \\
03 & 12,1 & 5,0 & 101 \\
04 & 11,3 & 4,8 & 130 \\
05 & 10,0 & 4,7 & 151 \\
06 & 9,2 & 4,6 & 167
\end{tabular}




\begin{tabular}{llll}
07 & 8,5 & 4,3 & 185 \\
08 & 7,9 & 4,0 & 197 \\
09 & 7,4 & 3,9 & 209 \\
10 & 7,0 & 3,8 & 220 \\
\hline
\end{tabular}

Na figura 3, pode-se verificar o comportamento dos sólidos solúveis totais ( ${ }^{\circ}$ Brix) do mosto durante a fermentação.

Figura 3 - Curva do decréscimo do teor de sólidos solúveis totais ( ${ }^{\circ}$ Brix) em função do tempo.

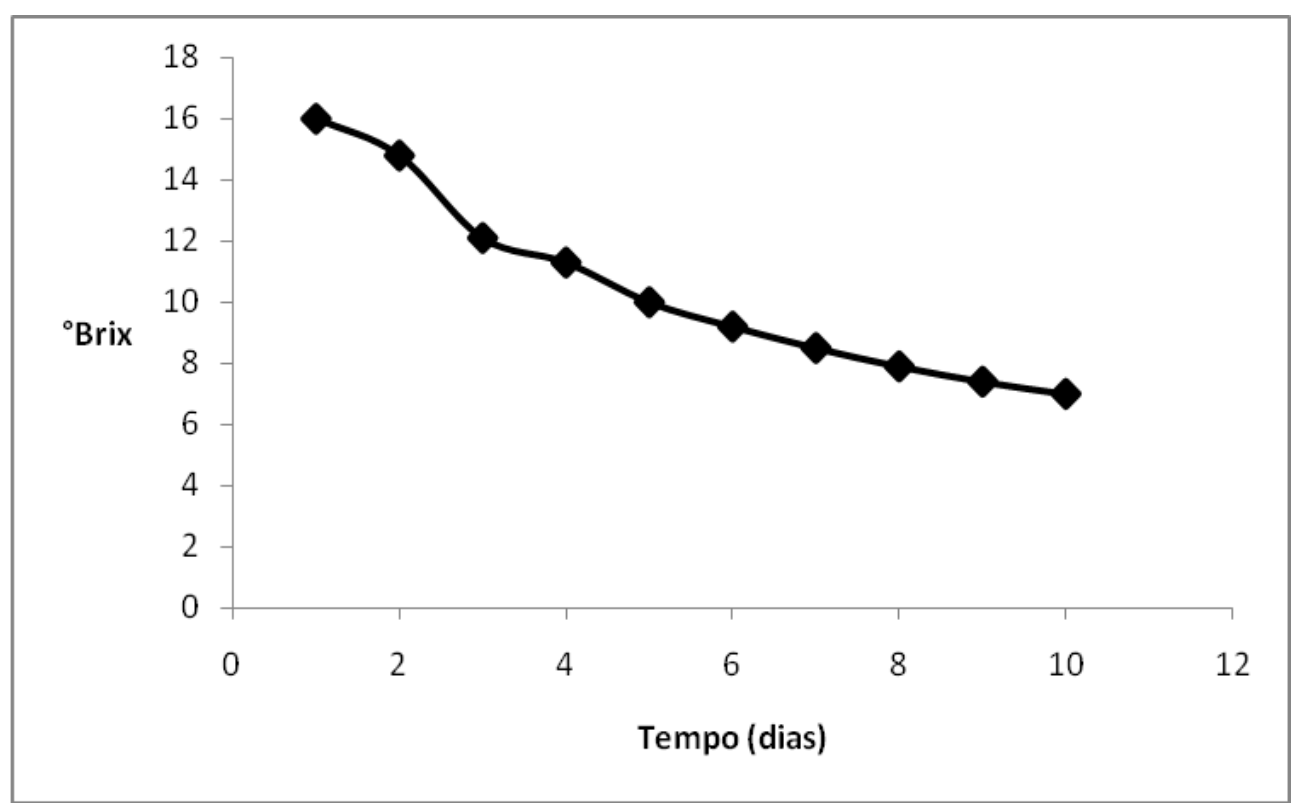

O fermentado de jaca obteve no final da fermentação um valor de 7,0 ${ }^{\circ}$ Brix, valor igual ao encontrado para o fermentado de laranja, (CORAZZA; RODRIGUES; NOZAKI, 2001); próximos para o fermentado de mangaba, 6,26 ${ }^{\circ}$ Brix (MUNIZ et al., 2002) e fermentado de mandacaru, 7,25 ${ }^{\circ}$ Brix (ALMEIDA et al., 2006).

Nos cinco primeiros dias houve um rápido consumo dos açúcares, fase tumultuosa da fermentação, fase pela qual há um grande desprendimento de gás carbônico, o desdobramento do açúcar inicia-se lentamente e vai aos poucos aumentando a intensidade (RIZZON; MIELE, 2002). Observa-se ainda que, no final da fermentação, o teor de sólidos solúveis tende a se permanecer constante. O decréscimo do teor de sólidos mostrado indica que ocorreu a conversão de açúcar em álcool.

Os comportamentos do pH e da Acidez estão expressos na Figura 4, estes dois parâmetros estão correlacionados, pois variações de $\mathrm{pH}$ influenciam na acidez do mosto.

Os valores do $\mathrm{pH}$ do mosto variaram de 5,8 a 3,8, constata-se que houve um decréscimo constante até o final da fermentação, em contrapartida, a acidez demonstrou crescente até o final da fermentação esses processos são decorrentes da produção de ácidos orgânicos, como ácido lático, acético e succínico. 
Figura 4 - Variação do pH e da Acidez em função do tempo de fermentação.

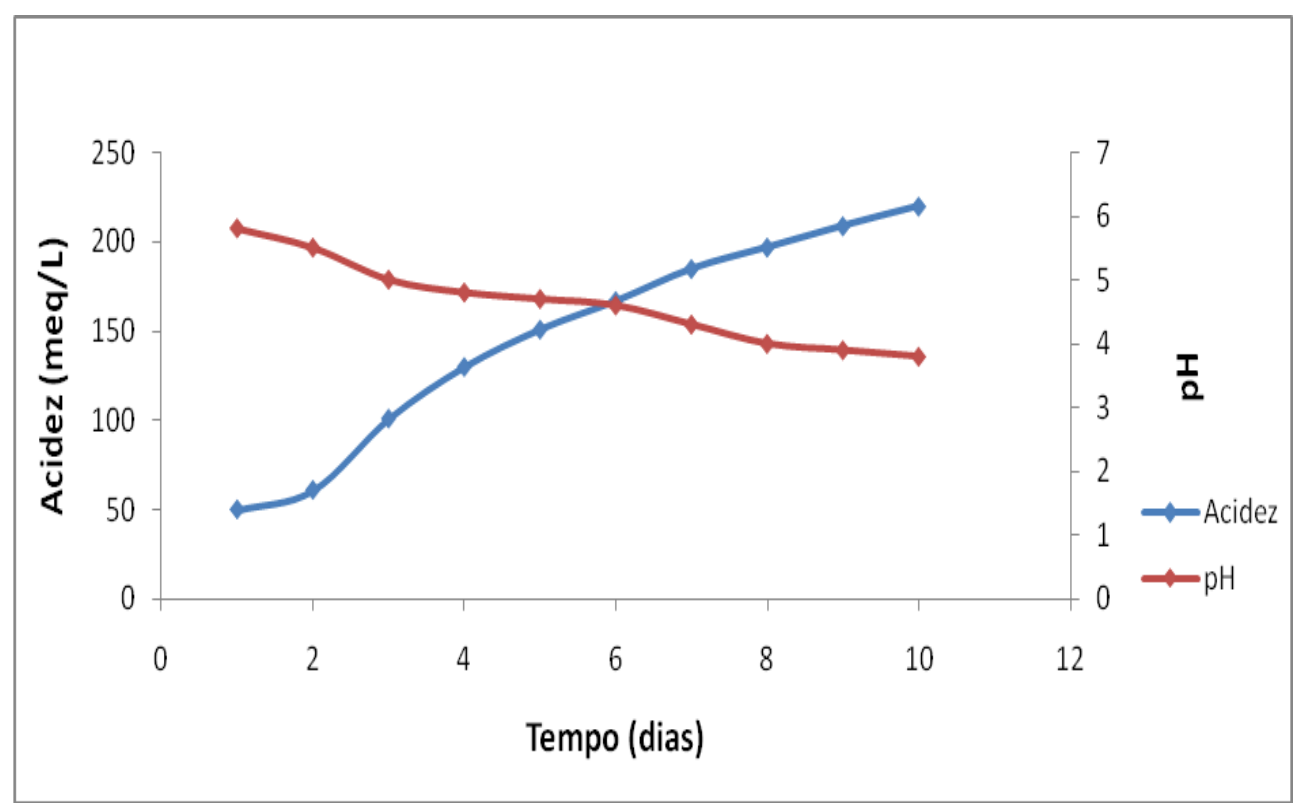

$\mathrm{O}$ aumento da acidez e redução do $\mathrm{pH}$ é encontrado em outros fermentados de frutas como no caso do fermentado de kiwi (BARTOLINI; SANT' ANNA; TORRES, 2001); fermentado de pupunha (ANDRADE; PANTOJA; MAEDA, 2003); fermentado de caju (ALMEIDA et al., 2006).

Segundo AQUARONE et al. (2001), com valores de pH com limites fixados no intervalo de 3 a 4, mais próximo do limite inferior, melhor será a resistência a bactérias que possam alterar as qualidades do fermentado. $\mathrm{O}$ pH do fermentado de jaca ficou no intervalo de 5,8 a 3,8, o que pode ser considerado um bom resultado, já que o pH no final da fermentação ficou em 3,8. Outros aspectos da fermentação tais como, crescimento de bactérias indesejáveis, efetividade do dióxido de enxofre, solubilidade de proteínas e reações de escurecimento também são influenciados pelo pH (MUNIZ et al, 2002).

Os fatores relacionados à acidez do fermentado têm participação importante nas características sensoriais e na estabilidade físico-química e biológica (RIZZON; MIELE, 2002). Na acidez pode-se constatar que houve um aumento constante do início até o final da fermentação, 50 a $220 \mathrm{meq} / \mathrm{L}$.

Os resultados obtidos nas análises físico-químicas da bebida alcoólica fermentada podem ser observados na tabela 2 .

Tabela 2 - Resultado das análises físico-químicas da bebida alcoólica fermentada de jaca.

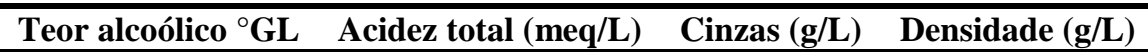


Segundo VOGT (1972), a reação de transformação do açúcar em álcool é uma reação importantíssima, onde, a quantidade de álcool produzida está diretamente ligada com a quantidade de açúcar presente no mosto. A bebida alcoólica fermentada de jaca, pode ser classificada como uma bebida alcoólica por apresentar teor alcoólico superior a $0,5{ }^{\circ} \mathrm{GL}$. Segundo a legislação brasileira, pode ser classificado como fermentado de fruta, pois se apresenta com graduação alcoólica dentro do intervalo de quatro a quatorze ${ }^{\circ} \mathrm{GL}$, a vinte graus Celsius (BRASIL, 1997).

Comparados aos estudos realizados com o fermentado alcoólico de jabuticaba, (SILVA et al., 2008), resultados próximos foram encontrados nos fermentados de jabuticaba nas safras de 2004 e 2005, onde alguns obtiveram teor alcoólico abaixo de 7,0 ${ }^{\circ} \mathrm{GL}$; e com a fermentação alcoólica do butiá com 6,0 de ${ }^{\circ} \mathrm{GL}$ (BERNADI et al., 2008). Comparando com outros fermentados de fruta, está muito abaixo. O fermentado de cajá obteve um valor de $12{ }^{\circ} \mathrm{GL}$ (DIAS; SCHWAN; LIMA, 2003); o fermentado de caju, $11,5^{\circ} \mathrm{GL}$ (TORRES NETO et al., 2006); o fermentado de laranja, 10,6 ${ }^{\circ} \mathrm{GL}$ (CORAZZA; RODRIGUES; NOZAKI, 2001); o fermentado de jamelão, 10,3 ${ }^{\circ} \mathrm{GL}$ (SOUZA; SILVA; TESHIMA, 2006); o fermentado de ciriguela, $10,0{ }^{\circ} \mathrm{GL}$; e o de mangaba, $9,8^{\circ} \mathrm{GL}$ (MUNIZ et al., 2002).

As cinzas representam os elementos minerais presentes no vinho e, geralmente, correspondem a aproximadamente $10 \%$ do extrato seco reduzido (RIZZON; MIELE, 2002). No caso de vinho de mesa comum branco, a legislação brasileira estabelece o mínimo de cinzas de 1,3 g/L (BRASIL, 1988). O valor encontrado para a bebida alcoólica fermentada de jaca foi de 3,55 g/L, valor semelhante se comparado ao do fermentado de jaca, 3,48 g/L (ASQUIERI; RABÊLO; SILVA, 2008). Mas se comparar os mesmos valores de cinzas como os encontrados por SANTOS et al (2005), para fermentado de acerola, que foi de 0,40 \pm 0,11, encontra-se diferença considerável, provavelmente por má fermentação ou pela presença de minerais estranhos da própria fruta.

Segundo VARNAM \& SUTHERLAND (1997), uma enorme quantidade de compostos contribuem, em maior ou menor medida, ao sabor e o aroma dos vinhos de frutas. Um conjunto de aldeídos, ésteres, cetonas, alcoóis, ácidos orgânicos e hidrocarbonetos contribuem para a formação do sabor. Então, o teor de acidez regula a aceitação do produto.

Em relação à acidez total, os valores da legislação para vinho de uva de mesa permitem um conteúdo mínimo de 55 meq/L e máximo de 130 meq/L (BRASIL, 1988). O valor encontrado na bebida alcoólica fermentada de jaca, para acidez total, foi de $220 \mathrm{meq} / \mathrm{L}$, muito elevado quando comparado ao fermentado de jaca, $100 \mathrm{meq} / \mathrm{L}$ de (ASQUIERI; RABÊLO; SILVA, 2008), ao fermentado do doce de jabuticaba, 106,3 meq/L (ASQUIERI et al., 2004), mas semelhante ao encontrado para fermentado de jabuticaba, $210 \mathrm{meq} / \mathrm{L}$ (CHIARELLI; NOGUEIRA; VENTURINI FILHO, 2005) e próximos aos de Silva (2008), que obteve valores de acidez muito elevados dos diferentes fermentados alcoólicos de jabuticaba de varias safras, onde a maioria ficou por volta dos 
$185 \mathrm{meq} / \mathrm{L}$. O valor encontrado pode ter sido influenciado por um crescimento indesejado e excessivo de bactérias que produzem acido acético, visto que essas se encontram mais adaptadas pras condições de produção da bebida fermentada (VARNAM e SUTHERLAND, 1997).

A densidade a $20{ }^{\circ} \mathrm{C}$ do fermentado de jaca foi de 0,99401. Valores próximos foram encontrados para fermentado de Kiwi, 0,9973 ${ }^{\circ} \mathrm{GL}$, (PAZ, 2007) de jabuticaba seco e doce com um ano de armazenamento, de 1,003 e 1,029 ${ }^{\circ} \mathrm{GL}$, respectivamente (ASQUIERI, 2004). A menor densidade obtida foi em decorrência do processo de filtração realizado na polpa e na bebida já fermentada.

Com relação ao índice de aceitação, a bebida se mostrou situada entre os termos "nem gostei / nem desgostei" (5) e "gostei ligeiramente" (6), a porcentagem de aceitação pelos provadores foi de $61 \%$, expresso na Tabela 3.

Tabela 3 - Caracterização sensorial da bebida alcoólica fermentada de jaca.

\begin{tabular}{ccc}
\hline Produto & Índice de aceitação & \% de aceitação \\
\hline Bebida alcoólica & 5,49 & 61 \\
fermentada de jaca & & \\
\hline
\end{tabular}

A bebida apresentou uma coloração amarela, proveniente possivelmente das partículas sólida remanescentes da fruta, isso se deve principalmente a técnica aplicada na filtração, através do auxílio de tecidos de algodão e crivo. $\mathrm{O}$ aroma da bebida apresentou características da fruta jaca. Quanto ao sabor a elevada acidez, que pode indicar o crescimento de microrganismos indesejáveis após a elaboração, comprometendo a qualidade sensorial da bebida. $\mathrm{O}$ fato, de a acidez ter sido bastante elevada chama atenção para o processamento, visto que esse comprometimento surtiu diretamente sobre o índice de aceitação final do produto.

A Figura 5 indica a relação entre a escala de pontuação e o percentual de provadores que atribuiu determinada nota a bebida alcoólica fermentada de jaca.

Figura 5 - Relação entre a escala de pontuação pelo percentual de provadores.

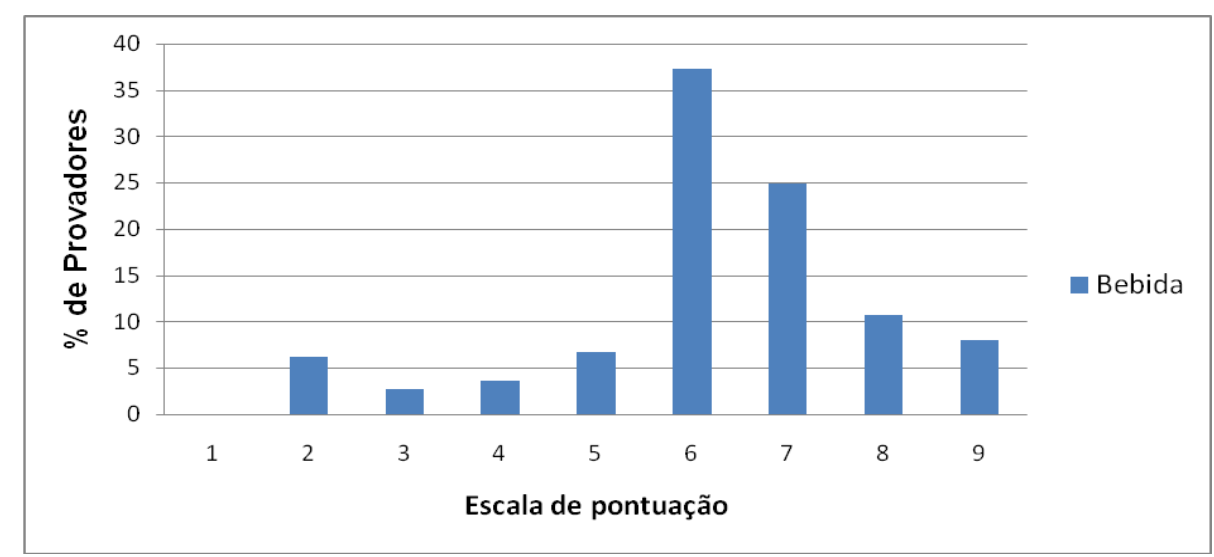

O índice de aceitação da bebida foi de 5,49 um resultado que chegou próximo aos encontrados por Muniz et al. (2002) com as bebidas fermentadas de ata, de ciriguela, de mangaba, obtendo índice de aceitação de 5,1, 4,3, 5,7, respectivamente. 
A frequência das notas varia entre os termos "desgostei muitíssimo" (1) a "gostei muitíssimo" (9), o termo "desgostei muitíssimo" não foi escolhido por nenhum dos 41 provadores (Figura 5). O termo com maior escolha foi o termo gostei ligeiramente (6).

\title{
4. Conclusões
}

A partir das análises físico-químicas da bebida alcoólica fermentada de jaca, observou-se que o produto se adéqua perfeitamente à legislação vigente de fermentados de frutas. Para obter maiores índices de teor alcoólico, seria necessário aumentar o número de dias de fermentação visto que a quantidade de açúcares está diretamente relacionada à quantidade de álcoois produzidos.

Os resultados da análise sensorial demonstraram que a jaca é apropriada para a produção de bebida alcoólica fermentada, porém, ainda necessita de maiores estudos à condução do processo fermentativo da jaca para obtenção de um produto dentro de um padrão aceitável pelo mercado. Percebe-se também que as técnicas empregadas influenciaram diretamente quanto ao índice de aceitação do produto, visto que houve uma possível contaminação por microorganismos que produzem acido acético, resultando em valores elevados de acidez do produto.

\begin{abstract}
The jackfruit (Artocarpus heterophyllus) produces most of all fruit grown and a large potential to be exploited industrially. Fermented beverages are fruit products with promising perspective of consumption. The aim of this study was to obtain an alcoholic beverage fermented jackfruit, and to determine the physicochemical characteristics of the drink and to evaluate the acceptance thereof. The liquor was performed using jackfruit variety of soft, sodium metabisulfite and dry yeast (Saccharomyces cerevisiae). In preparing the drink, the must was inoculated with yeast and fermented between 25 and $30^{\circ} \mathrm{C}$. The sulphite was performed in $90 \%$ of the wort, the sodium metabisulfite, was added at a rate of $200 \mathrm{mg} / \mathrm{L}$. The inoculation was performed by the method of foot-and-tub, made up the monitoring of fermentation performing physical-chemical analysis, which followed the Standards of the Ministry of Agriculture (BRASIL, 2005) such analysis occurred over a period of 10 days. From the final product were analyzed for total acidity ( $220 \mathrm{meq} /$ $\mathrm{L})$, ash $(3.55 \mathrm{~g} / \mathrm{L})$, density $(0.99401 \mathrm{~g} / \mathrm{L})$ and alcohol $\left(4.1^{\circ} \mathrm{GL}\right)$. The results of sensory analysis, using the test with acceptable hedonic scale, showed that the brew had an acceptance rate of $61 \%$, showing similar results with the fermentation of other tropical fruits. The results of sensory analysis showed that the jackfruit is suitable for the production of fermented alcoholic beverage, however, requires further studies to conduct the fermentation process to obtain the product within an acceptable standard by the market.
\end{abstract}

Key-words: alcoholic beverage, fermented fruit; jackfruit.

\section{Referências}

AQUARONE, E.; LIMA, U. A.; BORZANI, W. Biotecnologia: alimentos e Bebidas produzidos por fermentação. São Paulo: Edgar Blugher Ltda., 1983. 
ALMEIDA, M. M.; TAVARES, D. P. S. A.; ROCHA, A. S.; OlIVEIRA, L. S. C.; SILVA, F. L. H.; MOTA, J. C. Cinética da produção do fermentado do fruto do mandacaru. Revista Brasileira de Produtos Agroindustriais. Campina Grande, v. 8, n. 1, p. 35-42, 2006.

ANDRADE, J. S.; PANTOJA, L.; MAEDA, R. N. Melhoria do rendimento e do processo de obtenção da bebida alcoólica de pupunha (Bactris gasipaes Kunth). Ciência e Tecnologia de Alimentos, Campinas, v. 23 (supl.), p. 34-38, 2003.

ASQUIERI, E. R.; DAMIANI, C. CANDIDO, M. A.; ASSIS, E. M. Vino de jabuticaba (Mirciaria cauliflora Berg): Estudio de las características físico-químicas y sensoriales de los vinos tinto seco y dulce, fabricados con la fruta integral. Alimentaria (Madrid), n. 355, p. 111-121, 2004. DOI: 10.1590/S0101-20612008000400018.

ASQUIERI, E. R.; RABÊLO, A. M. S.; SILVA, A. G. M.. Fermentado de jaca: estudo das características físicoquímicas e sensoriais. Ciência e Tecnologia de Alimentos, v. 28, n. 4, p. 881-887, 2008.

BARTOLINI, F.; SANT’ ANNA, E. S.; TORRES, Regina Coeli. Comportamento das fermentações alcoólicas e acética de sucos de Kiwi (Actinidia deliciosa); composição dos mostos e métodos de fermentação acética. Ciência e Tecnologia de Alimentos, v. 21, n. 2, p. 236-243, 2001.

BRASIL. Decreto no 2.314, de 04 de setembro de 1997. Regulamenta a Lei No 8.918, de 14 de julho de 1994 , que dispõe sobre a padronização, a classificação, o registro, a inspeção, a produção e a fiscalização de bebidas. Brasília (DF). Ministério da Agricultura e do Abastecimento, disponível em: <http://www.receita.fazenda.gov.br/legislacao/Decretos/Ant2001/Ant1999/Dec231497.htm>. Acesso em: 14/03/2010.

BRASIL. Ministério da Agricultura, Pecuária e Abastecimento. Instrução Normativa $\mathbf{N}^{\mathbf{o}} 24$ de 08 de setembro de 2005. Aprova o Manual Operacional de Bebidas e Vinagres, disponível em: <http://www.agricultura.gov.br/portal/page?_pageid=33,1040532\&_dad=portal\&_schema=PORTAL>. Acesso em: $11 / 03 / 2010$.

BRASIL. Ministério da Agricultura, Pecuária e Abastecimento. Portaria n. 229, de 25 de outubro de 1988. Complementação dos padrões de identidade e qualidade do vinho, disponível em: <http://extranetagricultura.gov.br/sislegis-consulta/consultarLegislacao.do?operacao=visualizar\&id=3849>. Acesso em: $15 / 03 / 2010$.

CHIARELli, R. H. C.; NOGUEIRA, A. M. P.; VENTURINI FILHO, W. G. Fermentados de jabuticaba (Myrciaria cauliflora Berg): processos de produção, características físico-químicas e rendimento. Brazilian Journal of the Food Technology. v. 8, n. 4, p. 277-282, 2005.

CORAZZA, M. L., RODRIGUES, D. G. NOZAKI, J. Preparação e caracterização do vinho de laranja. Química Nova, v. 24, n. 4, p. 449-452, 2001.DOI: 10.1590/S0100-40422001000400004.

CORREA, M. P. Dicionário das plantas úteis do Brasil e das exóticas cultivadas. v. 6. Rio de Janeiro (RJ): Ministério da Agricultura/IBDF, 1984.

DIAS, D. R.; SCHWAN, R. F.; LIMA, L. C. O. Metodologia para Elaboração de Fermentado de Cajá (Spondias monbin L.). Ciência e Tecnologia de Alimentos, v. 23, n. 3, p. 342-350, 2003.DOI: 10.1590/S010120612003000300008.

FERREIRA, V. L. P. et al. Análise Sensorial:testes discriminativos e afetivos. Campinas. São Paulo (SP): SBCTA, 2000

GAVA, A. J. Princípios de tecnologia de alimentos. 7. ed. São Paulo: Nobel, 1984

GOMES, P. Fruticultura Brasileira. São Paulo: Nobel. 2007.

LIMA, L. F.; HESPANHOL, D. A.; SOUZA, M. C. Análise centesimal da torta de jaca - jacalícia. $6^{\circ}$ Simpósio de Ensino de Graduação. Universidade Metodista de Piracicaba - UNIMEP. 2008.

LUNA, J. V. U. Produção de mudas de fruteiras tropicais. Salvador (BA): EBDA. (Circular Técnica, 5), 1997.

MUNIZ, C. R.; BORGES, M. de F.; DE ABREU, F. A. P.; NASSU, R. T.; DE FREITAS, C. A. S. Bebidas fermentadas a partir de frutos tropicais. Boletim do CEPPA, v. 20, n. 2, p. 309-322, 2002. 
PAZ, M. F.; SCARTAZZINI, L. S.; OGLIARI, T. C.; BURLIN, C. Produção e Caracterização do Fermentado Alcoólico de Actinidia deliciosa Variedade Bruno Produzido em Santa Catarina. In: XVI Simpósio Nacional de Bioprocessos, SINAFERM 2007 - Anais - CD Room. Curitiba, 2007.

PEREIRA, L. G; AZEVEDO, J. A. G; OLIVEIRA, L. S; BARREIROS D. C; FERREIRA, A. L; BRANDÃO; FIGUEIRESO, E. M. P. Composição bromatológica e cinética de fermentação ruminal in vitro da jaca dura e mole (Artocarpus heterophyllus). Livestock Research for Rural Development. v. 19, n. 45, 2007.

RIZZON, L. A.; MIELE, A.. Avaliação da CV. Cabernet Sauvignon para elaboração de vinho tinto. Ciência e Tecnologia de Alimentos, v. 22, n. 2, p. 192-19, 2002. DOI: 10.1590/S0101-20612002000200015.

SANDHU, D. K.; JOSHI, V. K. Technology, quality and scope of fruitwines especialiy apple beverages. Indian Food Industry, v. 14, n. 1, p. 24-34, 1995.

SANTOS, S. C.; ALMEIDA, S. S.; TOLEDO, A. L.; SANTANA, J. C. C.; SOUZA, R. R. Elaboração e Análise Sensorial do Fermentado de Acerola (Malpighia Punicifolia L.). Braz. Journal Food Technology, $5^{\circ}$ SIPAL, 2005

SILVA, P. H. A.; FARIA, F. C.; TONON, B.; MOTA, S. J. D.; PINTO, V. T. Avaliação da composição química de fermentados alcoólicos de jabuticaba (Myrciaria jabuticaba). Química Nova, v. 31, n. 3, p. 595-600, 2008. DOI: 10.1590/S0100-40422008000300025.

SOUZA, L. C.; SILVA, W. L.; TESHIMA, E. Processo fermentativo do vinho de jamelão (Syzygium cumini fruit). In: XX Congresso Brasileiro de Ciência e Tecnologia de Alimentos, 2006, Curitiba. Resumos..., 2006.

TORRES NETO, A. B.; SILVA, M. E.; SILVA, W. B.; SWARNAKAR, R.; SILVA, F. L. H. Cinética e caracterização físico-química do fermentado do pseudofruto do caju (Anacardium occidentale L.). Química Nova, v. 29, n. 3, p. 489492, 2006. DOI: 10.1590/S0100-40422006000300015.

VARNAM, A. H.; SUTHERLAND, J. P. Bebidas: Teconlogía, química y microbiologia. Zaragoza: Acribia, 1997.

VIEIRA NETO, R. D. Frutíferas potencias para os tabuleiros costeiros e baixadas litorâneas. Aracaju: Embrapa Tabuleiros Costeiros / Empresa de Desenvolvimento Agropecuário de Sergipe-Emdagro, 2002.

VOGT, E. Fabricación de vinos. Departamento de Higiene y Tecnología de los Alimentos, Facultad de Veterinaria de León, Universidad de Oviedo. Editorial Acribia, Zaragoza, España, 1972.

\section{Dados completos dos autores:}

\begin{tabular}{|c|c|}
\hline $\begin{array}{l}\text { Nome completo: Erasto Ferreira de Assis Neto } \\
\text { Titulação: Tecnólogo Agroindustrial de } \\
\text { Alimentos } \\
\text { Endereço: Rua Ascendino Feitosa 74, Castelo } \\
\text { Branco III, João Pessoa, Paraíba, 58050-640. } \\
\text { Telefones para contato: (83) 3244-3417 / (83) } \\
\text { 9988-3562 e-mail: eras0205@yahoo.com.br }\end{array}$ & $\begin{array}{l}\text { Nome completo: Adriano César Calandrini } \\
\text { Braga. } \\
\text { Filiação institucional: Universidade do Estado } \\
\text { do Pará - UEPA } \\
\text { Departamento: Tecnologia de Alimentos } \\
\text { Titulação: Mestre } \\
\text { e-mail: adrianocalandrini@ hotmail.com }\end{array}$ \\
\hline $\begin{array}{l}\text { Nome completo: João Marcos Pinto da Cruz } \\
\text { Titulação: Tecnólogo Agroindustrial de } \\
\text { Alimentos } \\
\text { Endereço: completo para correspondência } \\
\text { (bairro, cidade, estado, país e CEP): Trav. Rita } \\
\text { de Cássia 1025, Triangulo, Santa Izabel do Pará, } \\
\text { Pará, 68790-000. } \\
\text { Telefones para contato: (91) 3744-4554 } \\
\text { e-mail: jmpc1025@hotmail.com }\end{array}$ & $\begin{array}{l}\text { Nome completo: João Hamilton Pinheiro de } \\
\text { Souza. - Orientador } \\
\text { Filiação institucional: Universidade do Estado } \\
\text { do Pará - UEPA } \\
\text { Departamento: Tecnologia de Alimentos } \\
\text { Titulação: Especialista } \\
\text { e-mail: jhpsouza@yahoo.com.br }\end{array}$ \\
\hline
\end{tabular}

